\title{
Photoacoustic characterization of custom-made thin film AlN MEMS ultrasound transducers
}

\author{
Jonas Kusch, Gordon M.H. Flockhart, Ralf Bauer, Deepak Uttamchandani \\ Centre for Microsystems \& Photonics, Department of Electronic \& Electrical Engineering \\ University of Strathclyde \\ Glasgow, UK \\ jonas.kusch@strath.ac.uk
}

\begin{abstract}
Two MEMS thin film aluminium nitride ultrasound transducer array designs for photoacoustic sensing applications are presented. They are characterized using the pitch and catch technique and photoacoustic excitation. The results of both approaches are subsequently compared.
\end{abstract}

Keywords—ultrasound, MEMS, transducer, characterization, photoacoustics

\section{INTRODUCTION}

Photoacoustic (PA) imaging and sensing has attracted a lot of attention in biomedical applications. This is due to its potential in improved specificity and resolution when compared to using conventional ultrasound for imaging [1]. In the push towards miniaturized PA systems, different types of ultrasound transducers are available. These miniaturised ultrasound transducers include capacitive micro-machined ultrasonic transducers (cMUT) and micro ring resonators (MRR). In the former, a cavity is etched into a silicon substrate which releases a thin film on which metal is deposited and which acts as a capacitor whose capacitance changes with applied pressure [2]. In the latter, the pressure wave deforms a micro ring structure and the resulting changes in optical resonance modes can be measured [3]. Both these methods offer a broad bandwidth but need to be actively operated by either applying a high bias voltage $\mathrm{V}>70 \mathrm{~V}$ (cMUT) [2] or coupling narrow band laser light into the sensor (MRR) [3]. Piezoelectric ultrasound transducers do not require to be actively driven but rather generate a voltage due to a pressure wave induced deformation of the piezoelectric material. In the presented work we focus on the characterization of custom piezoelectric MEMS ultrasound transducers for applications in PA detection. These devices are of interest as they can lead to broad bandwidth transducers and have the potential for mass production with a reduction in $\operatorname{cost}[4]$.

\section{DESIGN AND FABRICATION}

A narrow-band and a multi-bandwidth MEMS transducer array were designed with targeted frequency responses in the low $\mathrm{MHz}$ range, as shown in Fig. 1. These low frequencies increase the sensing depth of the transducers, with depths of $>5 \mathrm{~cm}$ reported for $2.25 \mathrm{MHz}$ transducers as detectors in PA experiments [5]. Our MEMS transducer designs were simulated using ANSYS Modal (ANSYS Inc, USA). The narrow-band MEMS transducer array was designed to enable variations in the overall active area and with potential uses as a phased array in mind. Its simulated resonance frequency was at $2.81 \mathrm{MHz}$ in air. The multi-bandwidth design was designed to enable a broadband MEMS transducer chip by combining transducers of different diameters and corresponding different centre frequencies. Its simulated resonance frequencies in air were 0.68 MHz, 1.07 MHz, 1.71 MHz and 2.91 MHz. These designs were fabricated using a cost-efficient multi-user process (PiezoMUMPs offered by Memscap Inc). The disk shaped transducer elements consist of a $0.5 \mu \mathrm{m}$ thick aluminium nitride piezoelectric active layer and a $10 \mu \mathrm{m}$ thick silicon-on-insulator device layer, fully backside released from a $400 \mu \mathrm{m}$ handle wafer. The released membrane diameters ranged from $240 \mu \mathrm{m}$ to $500 \mu \mathrm{m}$.
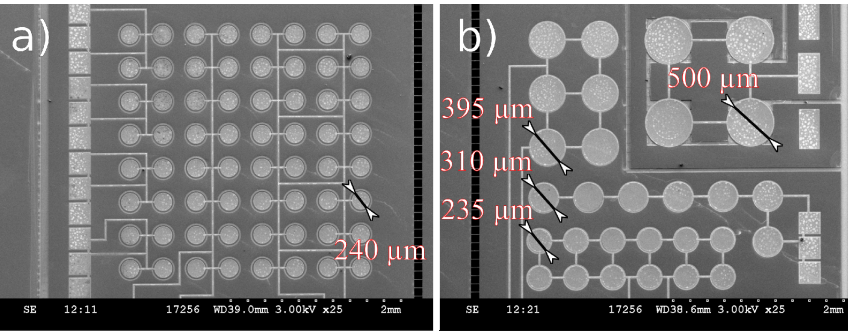

Fig 1: SEM images of the a) narrow-band transducer chip and b) the multi bandwidth transducer chip with diameters in $\mu \mathrm{m}$.

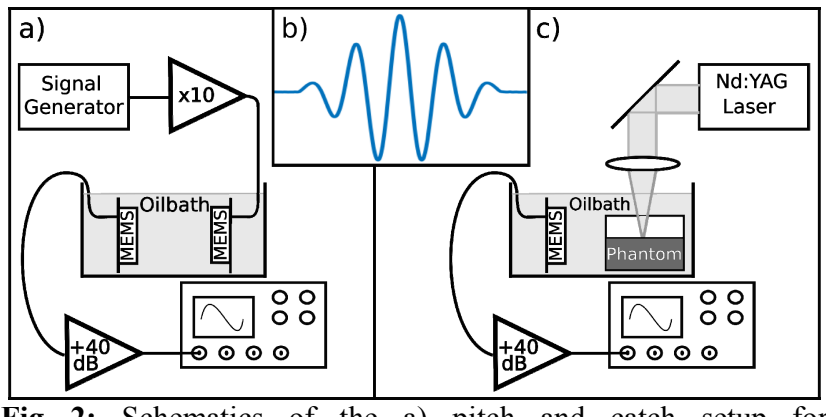

Fig 2: Schematics of the a) pitch and catch setup for characterization with b) the input waveform and c) the setup of the photoacoustic experiments

\section{METHODS \& RESULTS}

\section{A. Pitch \& catch technique}

The MEMS transducers were characterised using the pitch and catch technique (PCT). Here, two transducers of the same design were immersed in an oil bath $\left(\mathrm{v}_{1}=1450 \mathrm{~m} / \mathrm{s}\right)$ facing each other (see Fig. 2a). One transducer was actuated using a signal generator (Agilent, 33210A). Its output (see Fig. 2b) consisted of a train of five sinusoids at the actuation frequency $f_{A}$ overlaid with a Hamming window at a peak-to-peak voltage of $\mathrm{V}_{\mathrm{pp}}=70 \mathrm{~V}$. The signal output of the second MEMS transducer 
was amplified with a gain of $+40 \mathrm{~dB}$ (DHPVA-200, Femto) and recorded using a $1.5 \mathrm{GHz}$ oscilloscope (Agilent, Infiniium). It was filtered using a $6^{\text {th }}$ order Butterworth band-pass filter with cut-off frequencies at $1 / 3 f_{A}$ and $3 f_{A}$. The fast Fourier transform (FFT) was then applied to the signal and its frequency content of $f_{A}$ recorded. This was repeated over a range of $f_{A}$ resulting in the response spectrum. PCT experiments for the narrow-band MEMS transducer array show a centre frequency at $1.2 \mathrm{MHz}$ with a $-6 \mathrm{~dB}$ bandwidth of 0.58 $\mathrm{MHz}$ (see Fig. 3a). The characterization of the multi-bandwidth transducer array showed its centre frequencies at $0.2 \mathrm{MHz}, 0.4$ $\mathrm{MHz}, 0.62 \mathrm{MHz}$ and $1.25 \mathrm{MHz}$ with $-6 \mathrm{~dB}$ bandwidths of 0.11 $\mathrm{MHz}, 0.22 \mathrm{MHz}, 0.56 \mathrm{MHz}$ and $0.95 \mathrm{MHz}$ for diameters of 500 $\mu \mathrm{m}, 395 \mu \mathrm{m}, 310 \mu \mathrm{m}$ and $235 \mu \mathrm{m}$ respectively (see Fig. $3 \mathrm{~b}$ ).

\section{B. Photoacoustic excitation}

For characterization using PA excitation, the PA signal was generated using a $10 \mathrm{~ns}$ Nd:YAG laser (Brilliant B, Quantel) at $532 \mathrm{~nm}$ and a pulse repetition frequency of $10 \mathrm{~Hz}$ with pulse energies of $2.2 \mathrm{~mJ}$, focussed to a spot size of $250 \mu \mathrm{m}$ with a 70 $\mathrm{mm}$ lens onto a highly absorbing gelatine phantom (see Fig. 2c) ). The phantom consisted of two layers of $8 \%(\mathrm{~m} / \mathrm{m})$ gelatine, a clear layer and an absorbing layer. The latter was
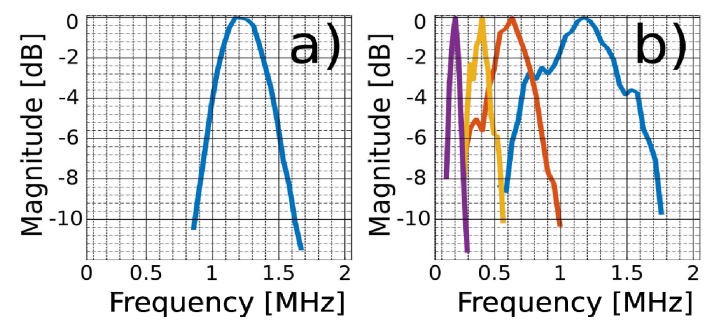

Fig 3: The bandwidth of the a) narrow-band transducer is 0.59 $\mathrm{MHz}$ with a centre frequency of $1.2 \mathrm{MHz}$ and $\mathrm{b}$ ) for the multibandwidth transducer array the centre frequencies are $0.2 \mathrm{MHz}$ (purple, $\mathrm{d}=500 \mu \mathrm{m}$ ), $0.4 \mathrm{MHz}$ (yellow, $\mathrm{d}=395 \mu \mathrm{m}$ ), $0.62 \mathrm{MHz}$ (red, $\mathrm{d}=310 \mu \mathrm{m})$ and $1.25 \mathrm{MHz}$ (blue, $\mathrm{d}=235 \mu \mathrm{m}$ ).

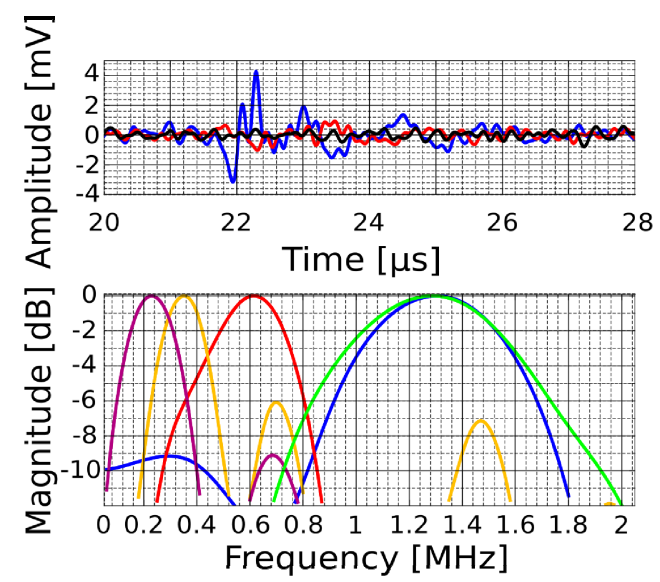

Fig 4: The PA pulses recorded in the absorbing layer (blue) \& the clear layer (red) and the background noise (black) (top). The centre frequencies (bottom) of the PA excitation results are at $1.25 \mathrm{MHz}$ for the narrow-band transducer (green) and for the multi-bandwidth array at $0.23 \mathrm{MHz}$ (purple, $500 \mu \mathrm{m}$ ), $0.36 \mathrm{MHz}$ (purple, $395 \mu \mathrm{m}$ ), $0.61 \mathrm{MHz}$ (purple, $310 \mu \mathrm{m}$ ) and $1.3 \mathrm{MHz}$ (purple, $235 \mu \mathrm{m}$ ) made absorbent by adding $0.4 \%(\mathrm{~m} / \mathrm{m})$ India ink. Both the gelatine phantom and the MEMS transducer were immersed in oil. PA pulses generated in the absorbing layer, the clear layer and the background noise (with the laser beam blocked) were recorded in Fig 4 top. A strong signal with a peak-to-peak voltage of $\mathrm{V}_{\mathrm{pp}}=8.5 \mathrm{mV}$ was recorded for the highly absorbing target and a weak one which is just above the noise level with a peak-to-peak voltage of $\mathrm{V}_{\mathrm{pp}}=1.5 \mathrm{mV}$ for the clear layer. The bandwidths of the narrow-band and the multi-bandwidth transducer arrays were experimentally determined by calculating the FFT of the recorded PA pulses. For the narrow -band design the centre frequency was measured as $1.25 \mathrm{MHz}$ and the $-6 \mathrm{~dB}$ bandwidth to be $0.89 \mathrm{MHz}$ (see Fig. 4 bottom, green). The evaluation PA pulses recorded with the multibandwidth transducer array showed that centre frequencies at $0.23 \mathrm{MHz}, 0.36 \mathrm{MHz}, 0.61 \mathrm{MHz}$ and $1.3 \mathrm{MHz}$ with $-6 \mathrm{~dB}$ bandwidths of $0.26 \mathrm{MHz}, 0.24 \mathrm{MHz}, 0.44 \mathrm{MHz}$ and $0.66 \mathrm{MHz}$ for diameters of $500 \mu \mathrm{m}, 395 \mu \mathrm{m}, 310 \mu \mathrm{m}$ and $235 \mu \mathrm{m}$, respectively as shown in Fig. 4, bottom.

\section{DISCUSSION AND CONCLUSION}

The centre frequency of the narrow-band transducers as measured with PCT is $1.2 \mathrm{MHz}$ and agrees well with the PA excitation results of $1.25 \mathrm{MHz}$. For the multi-bandwidth MEMS transducer array the results measured with PCT 0.2 $\mathrm{MHz}, 0.4 \mathrm{MHz}, 0.62 \mathrm{MHz}$ and $1.25 \mathrm{MHz}$ agree well with the centre frequencies calculated from the PA pulses with 0.23 $\mathrm{MHz}, 0.36 \mathrm{MHz}, 0.61 \mathrm{MHz}$ and $1.3 \mathrm{MHz}$ for diameters of 500 $\mu \mathrm{m}, 395 \mu \mathrm{m}, 310 \mu \mathrm{m}$ and $235 \mu \mathrm{m}$. These differ from the simulation results as simulations were done for air as a medium while the experiments were performed in oil which introduced additional dampening. The disparities in the measured transducer bandwidths are mostly due to the susceptibility of the transducer to noise at non-centre frequency actuation frequencies in PCT. In contrast, the PA excitation approach generates impulse-response like behaviour in the transducer due to the short-pulsed nature of the laser pulse.

\section{REFERENCES}

[1] P. Beard, "Biomedical photoacoustic imaging," Interface Focus, vol. 1, no. June, pp. 602-631, 2011.

[2] S. H. Pun, Y. Yu, J. Zhang, J. Wang, C. H. Cheng, K. F. Lei, Z. Yuan, and P. U. Mak, "Monolithic Multiband CMUTs for Photoacoustic Computed Tomography with In Vivo Biological Tissue Imaging," IEEE Trans. Ultrason. Ferroelectr. Freq. Control, vol. 65 , no. 3, pp. 465-475, 2018.

[3] H. Li, B. Dong, Z. Zhang, H. F. Zhang, and C. Sun, "A transparent broadband ultrasonic detector based on an optical micro-ring resonator for photoacoustic microscopy," Sci. Rep., vol. 4, 2014.

A. Hajati, D. Latev, D. Gardner, A. Hajati, D. Imai, M. Torrey, and M. Schoeppler, "Three-dimensional micro electromechanical system piezoelectric ultrasound transducer," Appl. Phys. Lett., vol. 101, no. $25,2012$.

[5] G. Ku and L. V. Wang, "Deeply penetrating photoacoustic tomography in biological tissues enhanced with an optical contrast agent," Opt. Lett., vol. 30, no. 5, p. 507, 2005. 